\title{
Doppler echocardiographic evaluation of pulmonary artery flow after modified Fontan operation: importance of atrial contraction
}

\author{
Shakeel Ahmed Qureshi, Roberto Richheimer, Roxane McKay, Robert Arnold
}

\begin{abstract}
Doppler echocardiography was used to evaluate blood flow in the pulmonary artery in 14 patients 2 to 42 months (mean (SD) 17 (12) months) after a modified Fontan operation incorporating a direct atriopulmonary anastomosis. Preoperatively six patients had tricuspid atresia, six had a double inlet left ventricle, and two had pulmonary atresia with an intact ventricular septum. The postoperative rhythm was sinus in 11 patients, junctional in one, ventricular pacing in one, and atrioventricular sequential pacing in one. In one patient the Doppler trace was unsatisfactory for analysis. In all patients forward flow in the pulmonary artery had biphasic peaks related to both atrial and ventricular contraction. The mean (SD) peak flow velocity that was synchronous with atrial contraction was $80(30) \mathrm{cm} / \mathrm{s}$ and that synchronous with ventricular contraction was $74(23) \mathrm{cm} / \mathrm{s}$. The atrial contribution to total pulmonary artery flow, assessed by velocity-time integrals, varied between $22 \%$ and $73 \%$ (mean (SD) $45(14) \%$ ). In patients with tricuspid atresia the mean (SD) peak flow velocity with atrial contraction was $90(27) \mathrm{cm} / \mathrm{s}$ and that with ventricular contraction was mean (SD) $68(24) \mathrm{cm} / \mathrm{s}$. In patients with double inlet left ventricle the mean (SD) peak flow velocity was $67(36) \mathrm{cm} / \mathrm{s}$ with atrial contraction and $80(25) \mathrm{cm} / \mathrm{s}$ with ventricular contraction. The atrial contribution to total pulmonary blood flow in patients with tricuspid atresia was significantly higher $(53(11) \%)$ than in those with double inlet left ventricle (37 (14)\%).
\end{abstract}

Pulmonary artery flow after modified Fontan operation was biphasic and was related to both atrial and ventricular contraction. The atrial contribution to pulmonary blood flow is greater in patients with tricuspid atresia than in those with a double inlet left ventricle. The mechanism of the second peak related to ventricular contraction is unknown.

Several technical modifications of the original Fontan operation ${ }^{1}$ have been used for physiological correction of patients with tricuspid atresia and double inlet left ventricle. These include the incorporation of a subpulmonary ventricular chamber, ${ }^{23}$ valved conduits between the right atrium and pulmonary artery, vena caval valves, and direct non-valved atriopulmonary anastomosis. $^{4-6}$ There are conflicting reports on the importance of right atrial contraction, and therefore sinus rhythm, to the success of this operation. $^{7-9}$

We assessed pulmonary artery flow patterns by Doppler ultrasound and evaluated the contribution of atrial contraction to total pulmonary blood flow after a modified Fontan operation with a direct non-valved atriopulmonary anastomosis.

\section{Patients and methods}

We studied 14 (eight boys and six girls aged 55-175 months (mean (SD) 90 (35) months at operation) randomly selected patients who underwent successful modified Fontan operation between December 1983 and June 1987 at the Royal Liverpool Children's Hospital. The Doppler echocardiographic study was performed between 2 and 42 months (mean (SD) 17 (12) months) postoperatively and the age at the study varied from 60 to 184 months (107 (37) months).

Six patients had tricuspid atresia. Four of these had associated pulmonary or subpulmonary stenosis and one had an absent pulmonary valve with intact ventricular septum. All five patients with reduced pulmonary blood flow had undergone palliative systemic-pulmonary artery shunts. The remaining patient had banding of the pulmonary trunk to reduce excessive pulmonary blood flow in infancy. Six patients had double inlet left ventricle. Two of the three with pulmonary or subpulmonary stenosis had been palliated with systemic-pulmonary artery shunts while three with unrestricted pulmonary blood flow had previous banding of the pulmonary trunk. Two patients had pulmonary atresia with intact ventricular septum. Both of these had undergone previous systemic-pulmonary artery shunts.

A direct posterior non-valved atriopulmonary anastomosis was constructed between the undivided superior vena cava, right atrium, and roof of the left atrium and the pulmonary arteries in 12 of the patients. In two patients an anterior anastomosis was constructed between the right atrial appendage and the pulmonary trunk/right pulmonary artery. In seven patients, the pulmonary trunk was divided and passed behind the aorta at the time of the repair, in five it was ligated, and in two 
the trunk and the pulmonary valve were left in continuity with the right ventricle.

In five patients with double inlet left ventricle the atria were partitioned by an oblique baffle that directed the pulmonary venous blood through both atrioventricular valves into the left ventricle. In one patient the right atrioventricular valve was closed.

Eleven of the patients were in sinus rhythm, one was in junctional rhythm, one in ventricular paced, and one in atrioventricular sequentially paced rhythm.

\section{DOPPLER ECHOCARDIOGRAPHY}

Cross sectional and Doppler echocardiography was performed using an Irex Meridian system. Cross sectional echocardiography was used to establish the optimal plane for pulsed Doppler sampling of the pulmonary artery flow distal to the atriopulmonary anastomosis. A $3.5 \mathrm{MHz}$ phased array transducer with a pulsed Doppler frequency of $2 \mathrm{MHz}$ was used for the examination. The optimum flow profiles in the pulmonary artery were obtained usually from the apical 4-chamber view or along the left sternal border, angling the transducer until the best audio signal was heard and the best spectral Doppler signal was displayed.

The Doppler velocity flow profiles were recorded at a paper speed of $75 \mathrm{~mm} / \mathrm{s}$. An electrocardiogram and phonocardiogram were recorded simultaneously with the Doppler spectral display to time the flow events. The recordings were made during quiet respiration in supine patients. Atrial contraction was defined on the traces from the $P$ wave to the first heart sound on the phonocardiogram. The ventricular contraction (systole) was defined as the interval between the first and second heart sounds on the phonocardiogram. Peak velocities were measured as the modal peaks on the spectral traces. The traces were digitised around the modal velocities using the inbuilt software of the Irex Meridian system. The velocity waveform during atrial contraction was digitised and the velocity-time integral obtained in centimetres. This was separated from the flow during the remainder of the cardiac cycle by drawing a vertical line to the zero line at the beginning of the downstroke of the waveform of the atrial contraction. The total pulmonary blood flow was then similarly digitised to obtain the total velocity-time integral. No attempt was made to measure the atriopulmonary anastomotic diameter, which was assumed to be constant throughout the cardiac cycle. The atrial contribution to the total pulmonary flow was calculated as follows: $100 \times$ (velocity-time integral of atrial contraction $(\mathrm{cm})$, divided by velocity-time integral of total pulmonary flow $(\mathrm{cm})$ ). An average of at least five cardiac cycles was used for all measurements.

Doppler traces that were suitable for analysis were obtained in 13 of the 14 patients. The traces of one patient with pulmonary atresia and intact ventricular septum, described separately, were not included in the analysis because junctional rhythm precluded accurate timing of the atrial contraction. The patients were divided into two groups for the analysis; those with tricuspid atresia were compared (by unpaired $t$ tests) with those with a double inlet left ventricle.

\section{Results}

In all the patients forward flow in the pulmonary artery had two peaks. Diastolic forward flow started at the second heart sound and was usually of low velocity. This was considered to be due to passive venous return into the pulmonary artery. Augmentation of the forward flow velocities then occurred; this began with the $\mathbf{P}$ wave, reached its peak between the $P$ wave and the peak of the $R$ wave, and returned to the baseline at the onset of the first heart sound. A second peak was seen during ventricular systole between the first and second heart sounds. In patients with tricuspid atresia the peak velocity after atrial contraction was higher than that after ventricular contraction; in contrast, in patients with double inlet left ventricle the pattern was reversed (fig 1A and B). In one patient with junctional rhythm the $P$ wave was masked by the QRS complex. Peak forward flow in this patient occurred in early ventricular systole (due to atrial contraction) and a further peak occurred in ventricular diastole with passive venous filling (fig 2 ). In one patient with ventricular paced rhythm for postoperative complete heart block, there were, in addition to a dominant forward flow peak occurring with ventricular systole, separate peaks unrelated to ventricular systole and caused by atrial contraction (fig 3).

Peak flow velocity in the pulmonary artery synchronous with atrial contraction was

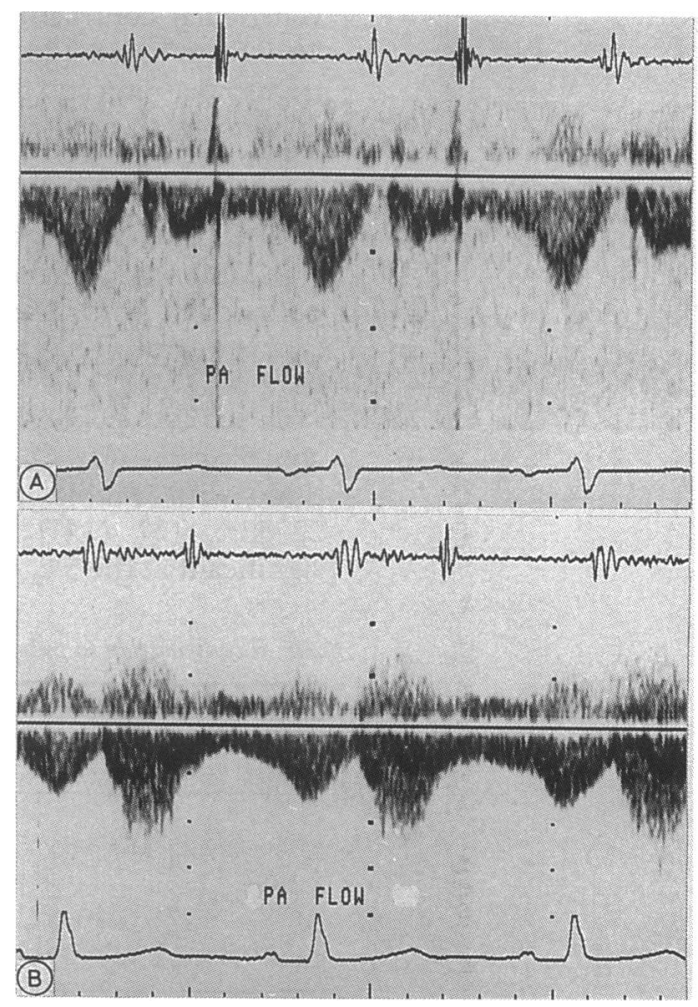

Figure 1 Doppler flow pattern in the pulmonary artery after Fontan operation showing biphasic flow in a patient with tricuspid atresia $(A)$ compared with the pattern obtained in a patient with double inlet left ventricle $(B)$. 
Figure 2 Doppler flow pattern in pulmonary artery in a patient with pulmonary atresia with intact ventricular septum in whom junctional rhythm developed after Fontan operation.

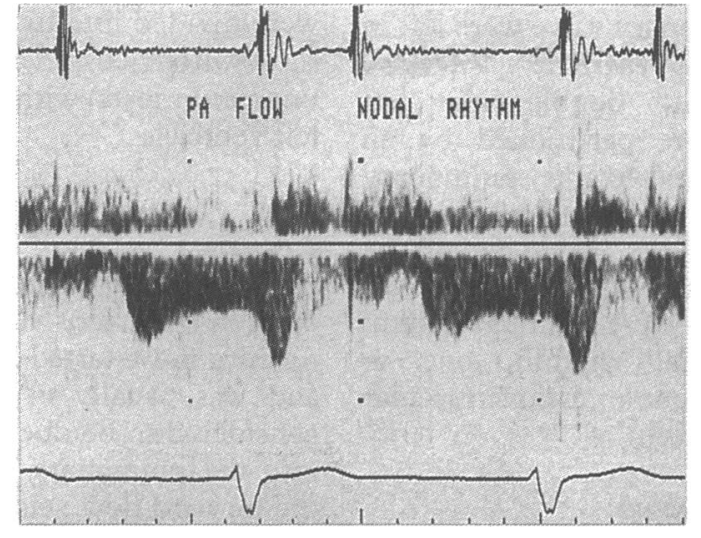

Figure 3 Doppler flow pattern in pulmonary artery in a patient with double inlet left ventricle in whom complete heart block developed after Fontan operation, and who had a ventricular pacemaker implanted (arrows indicate flow caused by atrial contraction).

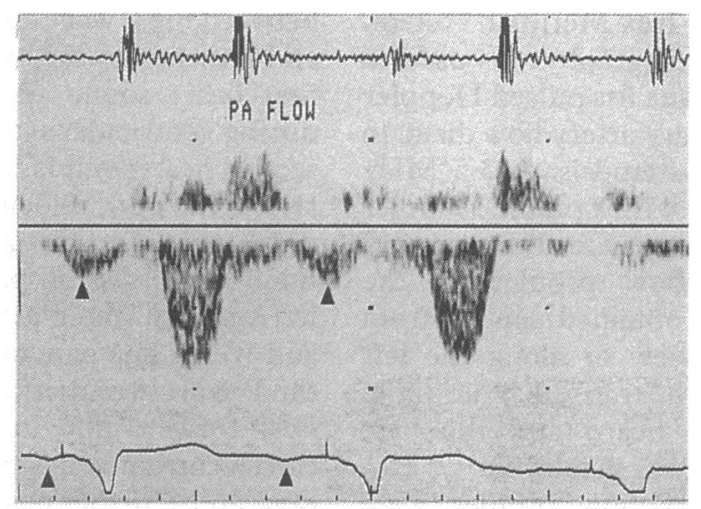

$27-123 \mathrm{~cm} / \mathrm{s}$ (mean (SD) $80(30) \mathrm{cm} / \mathrm{s}$ ), while that during ventricular contraction was 46-109 $\mathrm{cm} / \mathrm{s}(74(23) \mathrm{cm} / \mathrm{s})$. This difference was not significant at the $5 \%$ level.

In patients with tricuspid atresia the peak flow velocities during atrial contraction varied from 50 to $123 \mathrm{~cm} / \mathrm{s}(90(27) \mathrm{cm} / \mathrm{s})$, compared with $28-113 \mathrm{~cm} / \mathrm{s}(67(36) \mathrm{cm} / \mathrm{s})$ in those with double inlet left ventricle $(p=0 \cdot 17)$. During ventricular contraction peak flow velocity was $46-99 \mathrm{~cm} / \mathrm{s}(68(24) \mathrm{cm} / \mathrm{s})$ in patients with tricuspid atresia and $51-109 \mathrm{~cm} / \mathrm{s}(80(25) \mathrm{cm} / \mathrm{s})$ in those with double inlet left ventricle ( $p=$ $0.42)$.

\section{ATRIAL CONTRIBUTION}

The table shows the atrial contribution to total pulmonary flow assessed from the velocitytime integral. This varied between $22 \%$ and $73 \%(45(14) \%)$.

In patients with tricuspid atresia the atrial contribution was 41-73 (53 (11)\%) while in those with double inlet left ventricle it was $22-58 \%$ (37 (14)\%). This difference was significant at the $5 \%$ level.

Atrial contribution to pulmonary blood flow

\begin{tabular}{llllc}
\hline \multicolumn{2}{l}{ Tricuspid or pulmonary atresia } & & \multicolumn{2}{c}{ Double inlet left ventricle } \\
\cline { 5 - 6 } Case & $A C(\%)$ & & Case & $A C(\%)$ \\
\hline 1 & 41 & & $8 \dagger$ & 22 \\
2 & 43 & 9 & 26 \\
$3^{\star}$ & 48 & $10^{\dagger}$ & 27 \\
4 & 52 & 12 & 40 \\
5 & 55 & 13 & 49 \\
6 & 56 & & 58 \\
7 & 73 & & \\
\hline
\end{tabular}

*Before operation this patient had pulmonary atresia with intact ventricular septum.

tThis patient had an atrioventricular sequential pacemaker. This patient had a ventricular pacemaker.

AC, atrial contribution.
In one patient with pulmonary atresia and intact ventricular septum excision of the tricuspid valve and division of the pulmonary trunk were performed at the time of the modified Fontan operation to incorporate the hypoplastic right ventricle in the pulmonary circulation upstream to the atriopulmonary anastomosis. Doppler study showed that the atrial contribution to the total pulmonary blood flow was $48 \%$ and the flow pattern was similar to that in the patients with tricuspid atresia. Thus in this patient the incorporation of the hypoplastic ventricle did not affect the pulmonary flow pattern.

In two patients a hypoplastic right ventricle remained in continuity with the pulmonary circulation. One of them had pulmonary atresia with intact ventricular septum and a Waterston shunt. A transannular right ventricular outflow patch had been used to decompress the right ventricle. The hypoplastic tricuspid valve was closed at the time of the Fontan operation. When the Doppler study was carried out he was in junctional rhythm. The second patient had tricuspid atresia with an intact ventricular septum and no pulmonary valve. In both patients there was free regurgitation into the hypoplastic right ventricle.

The first patient had forward flow into the pulmonary artery during systole as a result of the atrial contraction, while the remaining forward flow occurred in mid and late diastole due to passive venous filling (fig 2). In the second patient Doppler flow traces were similar to those seen in patients with tricuspid atresia, the atrial contribution to the pulmonary flow being $41 \%$. Thus the inclusion of a subpulmonary ventricular chamber as a reservoir distal to the atriopulmonary anastomosis did not seem to affect the flow pattern in the pulmonary artery.

Two of the patients with double inlet left ventricle had atrial contributions to pulmonary blood flow of $49 \%$ and $58 \%$. Both these patients had higher preoperative mean right atrial pressures ( $>7 \mathrm{~mm} \mathrm{Hg}$ ) than the remaining patients with double inlet left ventricle.

\section{Discussion}

It has generally been accepted that sinus rhythm, and thus atrial contraction, is important for the success of the Fontan operation or its modifications, ${ }^{5}$ although controversy continues as to whether the success is due to an improved left ventricular output or to a direct contribution of the right atrium to the pulmonary blood flow. The value of including a subpulmonary ventricular chamber in the repair is also controversial ${ }^{2}$ and data from some recent studies suggest that atrial contraction may not be essential. ${ }^{810}$ Irdeed, in some complex defects, such as those in which the left atrioventricular valves are absent or hypoplastic or there are anomalies of venous drainage, total cavopulmonary connection has been proposed as an alternative to Fontan operation. ${ }^{11}$ Nevertheless, the original or modified Fontan operation will continue to be used in most patients with tricuspid atresia or double 
inlet left ventricle. To characterise the type of pulmonary artery blood flow after a direct nonvalved atriopulmonary anastomosis we studied the Doppler flow patterns in these patients.

It is difficult to evaluate atrial function by a reliable technique. Doppler echocardiography gives a qualitative and possibly semiquantitative assessment of atrial function, however, by measurement of the ratio of forward flow during atrial contraction to the total flow in the pulmonary artery. Nakazawa et al suggested that the role of atrial contraction after the Fontan operation may be more important in those patients with a smaller pulmonary artery index, irrespective of whether they have tricuspid atresia or double inlet ventricle. ${ }^{10}$ Conversely, in patients with a large pulmonary artery index, the right heart output may be less dependent on atrial contraction. These conclusions were based on an invasive study with a catheter mounted Doppler velocity probe. Volume flow is usually calculated from the mean velocity of flow and cross sectional area of the vessel where the flow velocity is measured. After the Fontan operation it is difficult to measure the area of the atriopulmonary anastomosis accurately. Because absolute values of volume flow may not be reliable, both we and Nakazawa $e t$ al have assumed this area to be constant throughout the cardiac cycle. ${ }^{10}$ We have further adapted the intracardiac Doppler methods of flow assessments to the more conventional transthoracic pulsed Doppler techniques.

Shemin and associates, in an experimental study, reported that the right atrium did not function as a pump in the maintenance of pulmonary blood flow after a right atrium to pulmonary artery conduit. ${ }^{12}$ Matsuda et al, in a study on dogs that had right atrium to pulmonary artery conduits with the right ventricle excluded from the pulmonary circulation, induced atrial fibrillation and found that both the systemic arterial pressure and pulmonary blood flow were maintained. ${ }^{8}$ With atrial fibrillation, however, pulsatile flow in the atriopulmonary conduit was lost. Nakazawa et al evaluated seven patients after a Fontan operation for tricuspid atresia. They used pulsed Doppler echocardiography, cardiac catheterisation, and intracardiac Doppler with a catheter tipped velocity transducer. ${ }^{7}$ They found that the flow patterns obtained by pulsed Doppler echocardiography were biphasic and identical to those obtained with a catheter tipped velocity transducer and that atrial contraction contributed to the forward flow of blood in the pulmonary artery. In a subsequent report, patients with a double inlet left ventricle were studied for comparison and similar flow patterns were obtained. ${ }^{10} \mathrm{DiSessa}$ et al also confirmed that the pulmonary artery flow was biphasic after the Fontan operation both in patients with tricuspid atresia and in those with double inlet left ventricle. ${ }^{9}$ The flow, however, was not evaluated quantitatively or semiquantitatively. Alboliras et al suggested that synchronised atrial contraction was not necessary for adequate cardiac output, despite finding acceleration in pulmonary artery flow during atrial systole by Doppler. ${ }^{13}$ Biphasic flow patterns in the pulmonary artery were reported by Hagler et al ${ }^{14}$ but, interestingly, in patients with reduced ventricular function the predominant flow occurred during ventricular systole.

Our measurements showed important differences between the patients with tricuspid or pulmonary atresia and double inlet left ventricle. The previous reports showed that the pulmonary artery flow was biphasic and these phases were related to atrial contraction and passive venous filling. In our study the second phase was consistently related to ventricular systole, which was followed by non-pulsatile low velocity flow caused by passive venous filling. These differences have not been emphasised before. We found that right atrial contraction, while contributing significantly to the total pulmonary blood flow in both groups of our patients, made a greater contribution to the pulmonary flow in patients with tricuspid atresia than in those with double inlet left ventricle. This may have been because higher atrial pressures cause greater right atrial hypertrophy. However, the mechanism of the second phase of flow that was synchronous with ventricular systole is not clear. DiSessa et al suggested that the second phase may have been the result either of right atrial relaxation and vena caval blood flowing into the pulmonary artery or filling of the left atrium from the pulmonary venous bed, which was in turn filled by flow from the pulmonary artery. ${ }^{9}$ However, our study showed that this flow occurred only during ventricular systole and was also pulsatile. This suggests "active" rather than "passive" flow into the pulmonary artery. In one of our patients with double inlet left ventricle, in whom ventricular function was impaired, the atrial contribution ( $58 \%$ ) to total pulmonary blood flow was higher than the rest of this group. While we found that Doppler echocardiography was useful in this study, it probably failed to detect slow and transient pulmonary regurgitation into the right atrium because of its very low velocity.

In conclusion, pulmonary artery flow after modified Fontan operation is biphasic. The atrial contribution to total pulmonary flow is greater in patients with tricuspid atresia than in those with a double inlet left ventricle. However, the importance of phasic pulmonary artery blood flow in the various subsets of patients and its long term effects on the pulmonary vasculature remain important questions for further investigation in a larger group of patients.

\footnotetext{
1 Fontan F, Baudet E. Surgical repair of tricuspid atresia. Thorax 1971;26:240-8.

2 Bull C, de Leval MR, Stark J, Taylor JFN, Macartney FJ. Use of a subpulmonary ventricular chamber in the Fontan circulation. J Thorac Cardiovasc Surg 1983;85:21-31.

3 Kreutzer G, Galindez E, Bono H, de Palma C, Laura JP. An operation for the correction of tricuspid atresia. $J$ Thorac Cardiovasc Surg 1973;66:613-21.

4 Kreutzer G, Vargas FJ, Schlichter AJ, et al. Atriopulmonary anastomosis. J Thorac Cardiovasc Surg 1982;83: 427-36.

5 Choussat A, Fontan F, Besse P, Vallot F, Chauve A, Bricaud $H$. Selection criteria for Fontan's procedure. In: Anderson RH, Shinebourne EA, eds. Paediatric cardiology. Edinburgh: Churchill Livingstone, 1977:559-66. 6 Yacoub MH, Radley-Smith R. Use of a valved conduit from
} 
right atrium to pulmonary artery for "correction" of single ventricle Circulation 1976;54:63-70.

7 Nakazawa M, Nakanishi T, Okuda $H$, et al. Dynamics of right heart flow after Fontan procedure. Circulation 1984, 69:306-12.

8 Matsuda H, Kawashima Y, Takavo H, Miyamoto K, Mori $T$. Experimental evaluation of atrial function in righ atrium-pulmonary artery conduit operation for tricuspid atresia. J Thorac Cardiovasc Surg 1981;81:762-7.

9 DiSessa TG, Child JS, Perloff JK, et al. Systemic venous and pulmonary arterial flow patterns after Fontan's and pulmonary arterial fow patterns after Fontan's ion 1984;70:898-902.

10 Nakazawa $M$, Nojima $K$, Okuda $H$, et al. Flow dynamics in the main pulmonary artery after the Fontan procedure in patients with tricuspid atresia or single ventricle. Circulat patients with tricuspid
ion $1987 ; 75: 1117-23$.
11 De Leval MR, Kilner P, Gewillig M, Bull C. Total cavopulmonary connection: a logical alternative to atriopulmonary connection for complex Fontan operations-experimental studies and early clinical experience. $J$ Thorac Cardiovasc Surg 1988;96:682-95.

12 Shemin RJ, Merrill WH, Pfeifer JS, Conkle DM, Morrow AG. Evaluation of right atrial-pulmonary artery conduits for tricuspid atresia. J Thorac Cardiovasc Surg 1979; 77:685-90.

13 Alboliras ET, Porter CJ, Danielson GK, et al. Results of the modified Fontan operation for congenital heart lesions in patients with preoperative sinus rhythm. $J$ Am Coll Cardiol 1985;6:228-33.

14 Hagler DJ, Seward JB, Tajik AJ, Ritter DG. Functional assessment of the Fontan operation: combined $M$-mode, assessment of the Fontan operation: combined M-mode, J Am Coll Cardiol 1984;4:756-64.

\section{VIEWS FROM THE PAST Louis $N$ Katz}

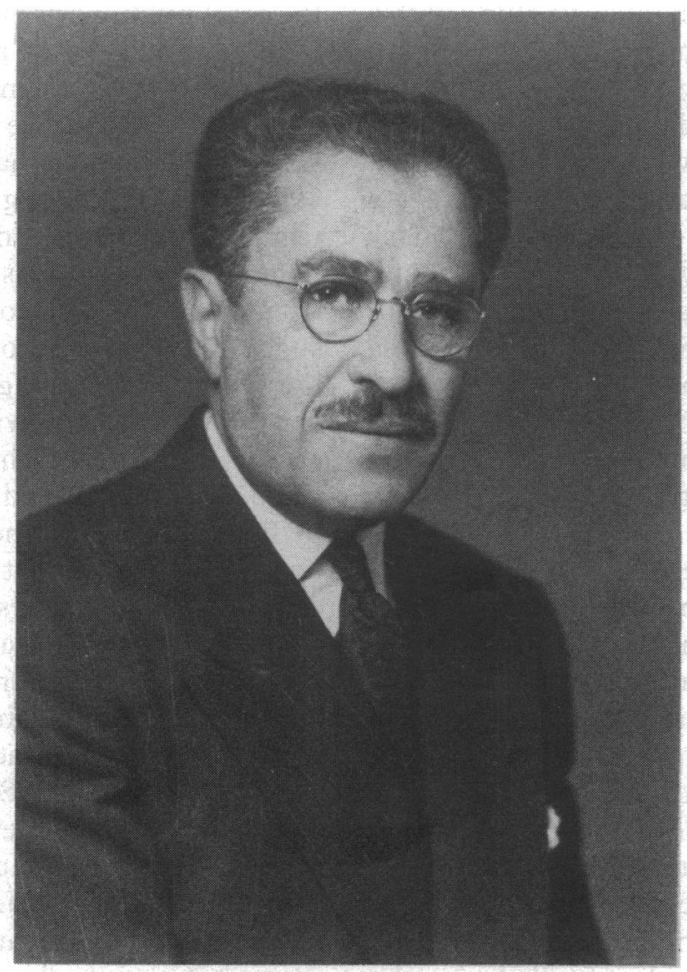

Louis N Katz (1897-1973) had a distinguished career as a physiologist in Carl Wiggers's department in Cleveland, Ohio. In 1924-25 he held a US National Research Council Fellowship at University College, London, in $\mathrm{E} \mathrm{H}$ Starling's department, where he studied myocardial metabolism with A V Hill (the future Nobel laureate). The two months he spent with Thomas Lewis had a key influence, for after he became the founding director of the Michael Reese Cardiovascular Institute in Chicago in 1930 he made it into a renowned centre of electrocardiography. (Photograph provided by Dr Arnold M Katz.)

DENNIS $M$ KRIKLER 